\title{
Photon Emission from Quark Gluon Plasma at RHIC and LHC
}

\author{
Poonam Jain, Yogesh Kumar \\ Department of Physics, Sri Aurobindo College, University of Delhi, New Delhi, India \\ Email: poonam.jn1@gmail.com, yogesh.du81@gmail.com
}

Received 5 April 2014; revised 1 May 2014; accepted 22 May 2014

Copyright (C) 2014 by authors and Scientific Research Publishing Inc.

This work is licensed under the Creative Commons Attribution International License (CC BY). http://creativecommons.org/licenses/by/4.0/

c) (i) Open Access

\begin{abstract}
We work on photon production through annihilation and Compton, and annihilation with scattering (AWS) processes through a quark-gluon plasma (QGP) in high energy nuclear collision incorporating the parametrization factors in strong coupling value and thermal dependent quark mass obtained through a coupling value. We find that photon yield increases with the increasing of the temperature and dominated by early times. We also compare the influence of coupling value on the spectrum of thermal photons. The production rate of photons is observed in the range of low and intermediate transverse momentum. The results are compared with other work.
\end{abstract}

\section{Keywords}

Direct Photon, Quark-Gluon Plasma

\section{Introduction}

There are many efforts attempted in the direction of new theoretical idea to study the formation of strongly interacting matter and quark-hadron phase transition at very high temperature and/or nuclear density. By colliding heavy-ions (such as $\mathrm{Au}$ on $\mathrm{Au}$ or $\mathrm{Pb}$ on $\mathrm{Pb}$ ) at the Relativistic Heavy-Ion Collider (RHIC) at Brookhaven National Laboratory (BNL) and the Large Hadron Collider (LHC) at Europian Organisation for Nuclear Research (CERN), expect to produce such a deconfined matter, called the Quark-Gluon Plasma (QGP) [1]-[13]. QGP is possibly the primordial fluid that permeated our universe when it was only a few microseconds old. In fact, the experiments at SPS/CERN, RHIC/BNL and LHC/CERN are trying to create such situation at high temperature for the study of QGP [14]. In these experiments, two massive nuclei are collided centrally and observed the formation of this dense nuclear matter. First indication for the discovery of the QGP in these experiments has been reported [15]-[17]. In order to identify the transient existence of the hot and dense quark-gluon system in heavy-ion collisions, electromagnetic probes, such as direct photons and dileptons have been suggested. They 
interact only electromagnetically, so called electromagnetic signals and their mean free paths are expected to be quite large than the transverse size of the collision volume created in any nuclear collision. As a result, highenergy photons produced in the interior of the plasma are not likely to suffer further collisions after they are produced and carry information directly from the reaction zone to the detector. Shuryak proposed photons as a promising direct probe [18] and they continue to be actively investigated [19]-[27]. Although, photons are considered as clean signals of QGP, there are few arguments arises due to massive pollution from lots of sources, like Drell-Yan processes, QGP, hadron gas, decay of charm particles etc. So we use these signals for extracting information directly from the collision zone. Hence, electromagnetic probes are considered to be ideal probes for the detection and study of subsequent evolution of QGP.

The investigations for photon production from quark-gluon plasma at finite temperature have been carried out [28]-[31]. An investigation of ideal fluid dynamics considers subsequent evolution of kinematically equilibrated QGP phase at very high nuclear density and temperature. Moreover experimental observations [32]-[34] from $\mathrm{Pb}-\mathrm{Pb}$ collision at $\sqrt{\mathrm{s}}=158$ AGeV by CERN WA80 and WA98 collaboration at SPS show interesting result in this electromagnetic probes [35]. These observations indicate the production of excess direct photons from the results of hadronic decays in the intermediate transverse momentum region of the most central collisions. Beside this, much other information confirms direct photon as a feasible signal for the formation and evolution of QGP. Many such active programs provide a platform to analyze the theoretical modeling of QGP evolution. In the theoretical modeling, the transverse momentum distribution function of direct photons is one significant factor in the photon induced reaction at the same energies $\sqrt{s}$ [36] [37] for a momentum greater than $1.5 \mathrm{GeV} / \mathrm{c}$ in the central collision. On this information, we focus on the measurement of photon emission which provides a good opportunity to study the early evolution of fireball. In RHIC, one might expect QGP is produced initially, it expands, cools and undergoes a phase transition when the temperature drops down to critical temperature $T=$ $0.17 \mathrm{GeV}$ and afterwards the system eventually freezes out into hadrons at the temprature $T=0.15 \mathrm{GeV}$ [38].

In this article, we consider a simple model of these strongly interacting matter "quark-gluon plasma". The matter is composed of a parametrized momentum factor in the quark mass. Due to this parametrization, the quark mass has a finite value and it removes the infrared (IR) divergence produced in the photon production [39]-[41]. The finite quark mass is defined as [42]:

$$
m_{q}^{2}=\frac{1}{6} \frac{N}{\ln \left[1+\frac{p^{2}}{\Lambda^{2}}\right]} T^{2}
$$

where $p=\left[\frac{\gamma N^{\frac{1}{3}} T^{2} \Lambda^{2}}{2}\right]^{\frac{1}{4}}$ known as minimum momentum cut off with $N=\frac{16 \pi}{\left[33-2 n_{f}\right]}$ and parametrization factor $\gamma^{2}=2\left[\frac{1}{\gamma_{q}^{2}}+\frac{1}{\gamma_{g}^{2}}\right]$ with $\gamma_{g}=6$ or $8 \gamma_{q}$ with $\gamma_{q}=1 / 6$. We calculate total photon spectra at different temperatures with parametrization factor for flavor 3 and compare the results with other work.

Thus, we organize the paper as: In Section 2, we present the total spectra in QGP and in Section 3, we show the results and at last Section 4, we give the conclusion.

\section{Photon Emission from QGP}

Thermal photons are ideal probes of quark-gluon plasma. The calculation of photon production from QGP is found to be very interesting theoretical problem. Moreover in range of the coupling parameter [43] [44] $\alpha_{s} \ll 1$, the output produces slow expansion near the equilibrium temperature, even though it is not justified condition. There are number of theoretical research works for production of photon from quark-gluon plasma. Most of the works are considered as quark-antiquark annihilation and Compton process. We focus on total photon spectra at different temperatures taking the photon production rate as a similar line of one loop calculation of Compton process $q(\bar{q}) g \rightarrow q(\bar{q}) \gamma$ plus annihilation $q \bar{q} \rightarrow g \gamma$ and the dominant contribution of $q \bar{q}$ annihilation with scattering obtained by Aurenche et al. known as AWS. 
The formulation of photon production with one loop order of Compton plus annihilation process is given by the expression [25] [28] [29] [45]-[48]

$$
E \frac{\mathrm{d} N}{\mathrm{~d}^{4} x \mathrm{~d}^{3} p}=\frac{1}{3 \pi^{2}} \alpha \alpha_{s} T^{2} \mathrm{e}^{-E / T}\left[\ln \left(\frac{4 E T}{2 m_{q}^{2}}\right)-C_{E}\right]
$$

where $C_{E}=1.415$.

Then the dominant contribution given by $q \bar{q}$ annihilation with scattering process known as AWS of production rate is given as [48]-[50]:

$$
E \frac{\mathrm{d} N}{\mathrm{~d}^{4} x \mathrm{~d}^{3} p}=\frac{16}{9 \pi^{5}} \alpha \alpha_{s} E T \mathrm{e}^{-E / T}\left(J_{T}-J_{L}\right)
$$

In the above expression, $J_{L}=-1.130$ and $J_{T}=1.20$ for quark flavor $n_{f}=3$. The strong coupling value is taken by Ref. [48].

Then we study the total photon spectrum at different temperatures by integrating the total rate over the spacetime history of the collision for all the processes after getting the temperature of the evolution from the model. We integrate rates at preferential temperatures, which are considered as temperature of transition to be completely hot phase. It is expressed as [46]-[48]:

$$
\frac{\mathrm{d} N}{\mathrm{~d}^{2} p_{T} \mathrm{~d} y}=\int \mathrm{d}^{4} x\left(E \frac{\mathrm{d} N}{\mathrm{~d}^{3} p \mathrm{~d}^{4} x}\right)=Q \int_{\tau_{0}}^{\tau_{f}} \tau \mathrm{d} \tau \int \mathrm{d} y\left(E \frac{\mathrm{d} N}{\mathrm{~d}^{3} p \mathrm{~d}^{4} x}\right)
$$

where $\tau$ is time evolution determined with the temperature from initial to final state with the rapidity $y_{\text {nuc }}= \pm 5.3$ corresponding to RHIC energy. $Q \sim 180 \mathrm{fm}^{2}$ is transverse cross section and $p_{T}$ is the photon transverse momentum. The quantity on the extreme R.H.S. is defined in the centre-of-mass system with the photon energy $E=p_{T} \cosh \left(y^{\prime}-y\right)$. We consider the system is dominated by the longitudinal expansion in the early stage in comparison to the transverse expansion [51] [52]. So we study photon yield for longitudinal expansion excluding the transverse. The approximation is suitable during the early stage where our interest is focused. Thus, with the values of rapidity and $p_{T}$, we get the total photon spectrum.

\section{Results}

In the results, we show total photon spectra from QGP through the different channels at finite temperature with the introduction of parametrization factors in strong coupling value and also in the quark mass. With a suitable choice of flow parameters for Compton scattering together with annihilation process and AWS, our calculation is performed for quark flavor $n_{f}=3$.

In Figure 1, we show total emission rate at temperature $T=0.35 \mathrm{GeV}$ with the variation of the photon transverse momentum for the process of Compton plus annihilation (C + Ann) and AWS. At closer inspection it turns out that the model calculation in the relevant range of photons from AWS process give the significant contribution and dominate over $\mathrm{C}+$ Ann process and show visible increment at temperature $T=0.35 \mathrm{GeV}$ of the QGP fireball. The results also give the enhancement as comparison to photon energy result. These observations are in good agreement with other theoretical work [25] [48]. The increase in the emission rate is highly effected by temperature of the system, and it seems to be large near creation of quark matter that is considered to be exist at very hot temperature. In Figure 2, we show photon spectra of $C+$ Ann process at different thermal temperatures with the coupling value and finite value of quark mass and also compare the results produced by [25] [48]. The interesting observation shows that the production rate is found to be very small increment than the other work up to temperature $T=0.31 \mathrm{GeV}$ and as we increases the temperature around $T=0.32 \mathrm{GeV}$, photon rate by Peitzmann et al. leads to a substantially higher rate as comparison to our result and more dominant for higher temperature. This is due to fact that the coupling constant $\alpha_{s}$ is temperature dependent and as temperature increases the value becomes smaller and it approaches the real system of deconfined QGP phase in which quarks and gluons are free and weakly interacting. At last in Figure 3, we calculate the same photon rate for AWS process for same values of temperature and compare the results with Refs. [25] [48]. The figure predicts that production rate as a function of transverse momentum has much enhancement over the production rate as function of photon energy. In both Figure 2, Figure 3, our model shows the enhancement up to $T=0.31 \mathrm{GeV}$ and 


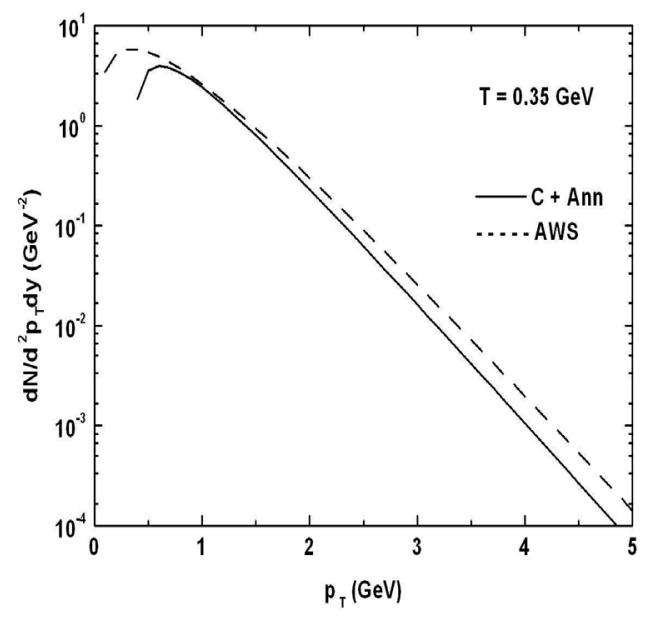

Figure 1. The total photon spectra through different channels at thermal temperature $T=0.35 \mathrm{GeV}$ for $n_{f}=3$.

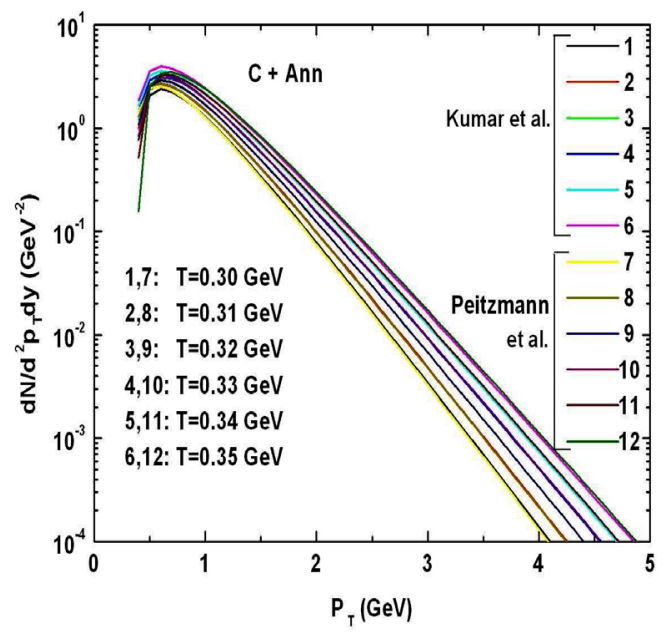

Figure 2. The total photon rate through Compton plus annihilation channel at different temperatures for $n_{f}=3$ and compared with other works.

further results are less sensitive to the higher temperature. Yet if we look at the production rate of the two processes, we found that AWS process shows better production rate in comparison to Compton plus annihilation process. This means that total photon spectra with the model of parametrization has a sizable enhancement over other theoretical calculation of photon spectra in all the possible processes with temperature up to $T=0.31 \mathrm{GeV}$. Our results also give the significant contribution over PHENIX data collaboration and results of Refs. [53]-[56].

\section{Conclusion}

We work on different sources for the photons and also discussed thermal photon production within a model and various sets of initial condition. We conclude that the calculation of total photon spectra as a function of transverse momentum incorporating the parametrization factors in strong coupling value and quark mass give the improved results in the photon yield products from the results [25] [48]. This implies that the consideration of parametrization factors in coupling value and thermal dependent quark mass has an important role in the photon measurements of the high-energy heavy-ion collisions. The QGP fireball with the parametrization factor gives a significant contribution and improved the calculation of photon radiation at temperature up to $T=0.31 \mathrm{GeV}$ afterwards our results are not sensitive at temperature more than $T=0.31 \mathrm{GeV}$ in both processes with flavor 3 . 


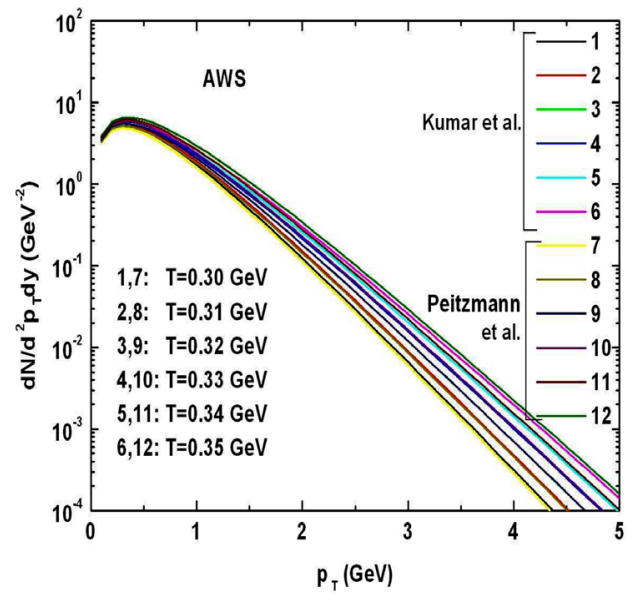

Figure 3. The total photon rate through AWS channel at different temperatures for $n_{f}=3$ and compared with other works.

Overall our model of parametrization plays an important and interesting role in photon emission and highly sensitive up to $T=0.31 \mathrm{GeV}$. Also our results enhance photon production rate over other work.

\section{Acknowledgements}

We thank S. Somorendro Singh and A. K. Jha for their critical constructive suggestions and discussions in preparing the manuscript.

\section{References}

[1] Arsene, I., et al. (2005) Nuclear Physics A, 757, 1-27. http://dx.doi.org/10.1016/j.nuclphysa.2005.02.130

[2] Adcox, K., et al. (2005) Nuclear Physics A, 757, 184-283. http://dx.doi.org/10.1016/j.nuclphysa.2005.03.086

[3] Back, B.B., et al. (2005) Nuclear Physics A, 757, 28-101. http://dx.doi.org/10.1016/j.nuclphysa.2005.03.084

[4] Adams, J., et al. (2005) Nuclear Physics A, 757, 102-183. http://dx.doi.org/10.1016/j.nuclphysa.2005.03.085

[5] Adare, A., et al. (2010) Physics Review C, 81, 034911. http://dx.doi.org/10.1103/PhysRevC.81.034911

[6] Adler, S.S., et al. (2007) Physics Review Letter, 98, Article ID: 012002. http://dx.doi.org/10.1103/PhysRevLett.98.012002

[7] Adare, A., et al. (2007) Physics Review Letter, 98, Article ID: 162301. http://dx.doi.org/10.1103/PhysRevLett.98.162301

[8] Adcox, K., et al. (2002) Physics Review Letter, 88, Article ID: 022301. http://dx.doi.org/10.1103/PhysRevLett.88.022301

[9] Adler, C., et al. (2002) Physics Review Letter, 89, Article ID: 092301. http://dx.doi.org/10.1103/PhysRevLett.89.092301

[10] Adler, S.S., Afanasiev, S., Aidala, C., et al. (2003) Physics Review Letter, 91, Article ID: 072301. http://dx.doi.org/10.1103/PhysRevLett.91.072301

[11] Chujo, T. (2003) Nuclear Physics A, 715, 151c-160c. http://dx.doi.org/10.1016/S0375-9474(02)01423-9

[12] Braun-Munzinger, P., Specht, H.J., Stock, R. and Stocker, H. (1996) Nuclear Physics A, 610, xi-xii.

[13] Geiger, K. and Müller, B. (1992) Nuclear Physics B, 369, 600-654. http://dx.doi.org/10.1016/0550-3213(92)90280-O

[14] QM01 (2002) Nuclear Physics A, 698.

[15] Gyulassy, M. (2004) The QGP Discovered at RHIC.

[16] Shuryak, E. (2004) What RHIC Experiments and Theory Tell Us about Properties of Quark-Gluon Plasma?

[17] Heinz, U. (2004) Thermalization at RHIC.

[18] Shuryak, E.V. (1978) Physics Letters B, 78, 150-153. http://dx.doi.org/10.1016/0370-2693(78)90370-2

[19] Arleo, F., Aurenche, P., Bopp, F., Dadic, I., David, G., Delagrange, H., et al. (2004) Photon Physics in Heavy Ion Col- 
lisions at the LHC.

[20] Shuryak, E.V. (1980) Physics Report, 61, 71-158. http://dx.doi.org/10.1016/0370-1573(80)90105-2

[21] Kajantie, K. and Miettinen, H.I. (1981) Zeitschrift fuer Physik C Particles and Fields, 9, 341-345. http://dx.doi.org/10.1007/BF01548770

[22] Domikos, G. and Goldman, J. (1981) Quark-Matter Diagonstics. Physics Review D, 23, 203. http://dx.doi.org/10.1103/PhysRevD.23.203

[23] Mclerran, L.D. and Toimela, T. (1985) Physics Review D, 31, 545-563. http://dx.doi.org/10.1103/PhysRevD.31.545

[24] Gale, C. and Kapusta, J.I. (1991) Nuclear Physics B, 357, 65-89. http://dx.doi.org/10.1016/0550-3213(91)90459-B

[25] Peitzmann, T. and Thoma, M.H. (2002) Physics Reports, 364, 175-246. http://dx.doi.org/10.1016/S0370-1573(02)00012-1

[26] Cassing, W. and Bratkovskaya, E.L. (1999) Physics Report, 308, 65-233. http://dx.doi.org/10.1016/S0370-1573(98)00028-3

[27] Aurenche, P. (2002) Photon Production in Heavy Ion Collisions. http://arxiv.org/abs/hep-ph/0201011

[28] Kapusta, J., Lichard, P. and Seibert, D. (1991) Physics Review D, 44, 2774-2788. http://dx.doi.org/10.1103/PhysRevD.44.2774

[29] Kapusta, J., Lichard, P. and Seibert, D. (1993) Physics Review D, 47, 4171. http://dx.doi.org/10.1103/PhysRevD.47.4171

[30] Baier, R., Nakkagawa, H., Niégawa, A. and Redlich, K. (1992) Zeitschrift fuer Physik C Particles and Fields, 53, 433438. http://dx.doi.org/10.1007/BF01625902

[31] Alam, J., Sarkar, S., Hatsuda, T., Nayak, T.K. and Sinha, B. (2001) Physics Review C, 63, Article ID: 021901.

[32] Aggarwal, M.M., Nilsson, P., Nishimura, S., Nomokonov, P., Nystrand, J., Obenshain, F.E., et al. (2000) Physics Review Letters, 85, 3595-3599. http://dx.doi.org/10.1103/PhysRevLett.85.3595

[33] Aggarwal, M.M., Ahammed, Z., Angelis, A.L.S., Antonenko, V., Arefiev, V., Astakhov, V., et al. (2004) Physics Review Letters, 93, Published Online.

[34] Albrecht, R., Antonenko, V., Awes, T.C., Barlag, C., Berger, F., Bloomer, M., et al. (1996) Physics Review Letters, 76, 3506-3509. http://dx.doi.org/10.1103/PhysRevLett.76.3506

[35] Morrison, D.P., Akiba, Y., Alford, O., Allen, M., Allen, W., Alley, G., et al. (1998) Nuclear Physics A, 638, 565c-569c. http://dx.doi.org/10.1016/S0375-9474(98)00390-X

[36] Wang, S.Y. and Boyanovsky, D. (2001) Physics Review D, 63, Article ID: 051702.

[37] Boyanovsky, D. and de Vega, H.J. (2003) Physics Review D, 68, Article ID: 065018.

[38] Singh, S.S. and Kumar, Y. (2013) Journal of Modern Physics, 4, 582-586. http://dx.doi.org/10.4236/jmp.2013.45082

[39] Pisarski, R.D. (1988) Nuclear Physics B, 309, 476-492. http://dx.doi.org/10.1016/0550-3213(88)90454-3

[40] Pisarski, R.D. (1989) Physics Review Letters, 63, 1129-1132. http://dx.doi.org/10.1103/PhysRevLett.63.1129

[41] Braaten, E. and Pisarski, R.D. (1990) Nuclear Physics B, 337, 569-634. http://dx.doi.org/10.1016/0550-3213(90)90508-B

[42] Kumar, Y. and Singh, S.S. (2012) Canadian Journal of Physics, 90, 955-961. http://dx.doi.org/10.1139/p2012-089

[43] Moore, G.D. (2004) Electromagnetic Emission and Energy Loss in the QGP.

[44] Ruuskanen, P.V. (1991) Quark-Gluon Plasma. World Scientific, Singapore.

[45] Traxler, C.T., Vija, H. and Thoma, M.H. (1995) Physics Letter B, 346, 329-334. http://dx.doi.org/10.1016/0370-2693(95)00004-5

[46] Renk, T. (2003) Physics Review C, 67, Article ID: 064901.

[47] Traxler, C.T. and Thoma, M.H. (1996) Physics Review C, 53, 1348-1352. http://dx.doi.org/10.1103/PhysRevC.53.1348

[48] Kumar, Y. and Singh, S.S. (2013) ISRN High Energy Physics, 2013, Article ID: 156747. http://dx.doi.org/10.1155/2013/156747

[49] Aurenche, P., Elis, F., Kobes, R. and Zaraket, H. (1998) Physics Review D, 58, Article ID: 085003.

[50] Steffen, F.D., and Thoma, M.H. (2001) Physics Letter B, 510, 98-106. http://dx.doi.org/10.1016/S0370-2693(01)00525-1

[51] Ollitrault, J.Y. (2008) European Journal of Physics, 29, 275. http://dx.doi.org/10.1088/0143-0807/29/2/010

[52] Martinez, M. and Strickland, M. (2008) Physics Review C, 78, Article ID: 034917.

[53] Adare, A., et al. (2010) Physics Review Letters, 104, Article ID: 132301. 
http://dx.doi.org/10.1103/PhysRevLett.104.132301

[54] Büsching, H.B. (2006) Nuclear Physics A, 774, 103-112. http://dx.doi.org/10.1016/j.nuclphysa.2006.06.033

[55] Gale, C., Turbide, S., Frodermann, E. and Heinz, U. (2008) Journal of Physics G: Nuclear and Particle Physics, 35, Article ID: 104119. http://dx.doi.org/10.1088/0954-3899/35/10/104119

[56] Dusling, K. and Zahed, I. (2010) Physics Review C, 82, Article ID: 054909. 